\title{
Visceral Manipulation Decreases Pain, Increases Cervical Mobility and Electromyographic Activity of the Upper Trapezius Muscle in Non-Specific Neck Pain Subjects with Functional Dyspepsia: Two Case Reports
}

\author{
Andréia C. O. Silva, PT, MSc,${ }^{1}$ Claudia S. Oliveira, PT, PhD,${ }^{1}$ Daniela A. Biasotto-Gonzalez, PT, \\ $\mathrm{PhD},{ }^{1}$ Marco A. Fumagalli, Eng, PhD, ${ }^{2}$ Fabiano Politti, PT, $\mathrm{PhD}^{1 *}$ \\ ${ }^{1}$ Postgraduate Program in Rehabilitation Sciences, Physical Therapy Department, Universidade Nove de Julho, São Paulo, \\ Brazil, ${ }^{2}$ Faculty of Mechanical Engineering, Faculdade das Américas, São Paulo, Brazil
}

Background and Purpose: The lack of clear knowledge about the etiology of nonspecific neck pain (NS-NP) strengthens the need for other mechanisms, still poorly described in the literature, to be investigated. Therefore, a quantitative analysis of two cases of NS-NP in subjects with functiona dyspepsia was conducted in order to verify the immediate and seven-day postintervention effects of visceral manipulation (VM) to the stomach and liver on neck pain, cervical range of motion (ROM), and electromyographic (EMG) activity of the upper trapezius muscle.

Case Description: Case A was an 18-year-old female with a complaint of nonspecific neck pain for one year, with reported pain on waking, momentary intermittent pain, and occasional symptoms of paresthesia in the upper limbs. Case $B$ was a 25-year-old female with a complaint of cervical pain for one year, accompanied by pain in the unilateral temporomandibular joint, and medial thoracic region. Both cases presented functional dyspepsia.

Outcomes: The results demonstrated (subjects $A$ and $B$, respectively) a general increase in cervical ROM (range: $12.5 \%$ to $44.44 \%$ ) and amplitude of the EMG signal (immediately postintervention: $\mathbf{5 7 . 6 2}$ and $\mathbf{2 0 . 7 8}$; post seven days: $53.54 \%$ and $18.83 \%$ ), and an increase in muscle fiber conduction velocity immediately postintervention $(4.44 \%$ and $7.44 \%)$ and a decrease seven days postintervention $(25.25 \%$ and $21.18 \%$ ). For pain, a decrease was observed immediately postintervention $(23.07 \%$ and $76.92 \%)$ and seven days postintervention $(100 \%$ for both subjects).

Discussion: A single VM provided important clinical improvement in neck pain, cervical spine range of motion, and EMG activity of the upper trapezius muscle, immediately and seven days postintervention in two NS-NP subjects with functional dyspepsia.

KEY WORDS: neck pain; muscle pain; functional dyspepsia; visceral manipulation; electromyography

\section{INTRODUCTION}

Non-specific neck pain (NS-NP) is characterized by pain from structures located in the region between the superior nuchal line and the spinal process of the first thoracic vertebra ${ }^{(1)}$ without association with any specific systemic disease.

Among etiological factors, poor posture, anxiety, depression, cervical tension, and occupational or sports activities are all mentioned, since the origin is often due to multifactorial and/or little known causes. ${ }^{(2)}$ Thus, the lack of clear knowledge about the etiology of NS-NP strengthens the need for other mechanisms, still poorly described in the literature, to be investigated. An example is the possibility that alterations in the mobility or functioning of the viscera found in the abdominal cavity may exert influences through mechanical and neural pathways in the cervical region.

One of the justifications for this relationship may be in possible medullary facilitation, at the cervical level, provided by afferent stimuli of the phrenic nerve. This nerve is responsible for sensory innervation of the subdiaphragmatic peritoneum, ${ }^{(3)}$ the coronary and falciform ligaments and capsule of the liver. ${ }^{(4)}$ Thus, mobility restriction of these tissues could generate nociceptive afferents at the cervical level which may lead to spinal cord facilitation between the cervical vertebrae $\mathrm{C} 3$ to $\mathrm{C}^{(3)}$ which would justify cervical pain caused by the alteration in visceral mobility.

Another possible visceral influence in the cervical region may be the anatomic relationship ${ }^{(5)}$ between the accessory nerve, which innervates the sternocleidomastoid and upper trapezius (UT) muscles, and the vagus nerve, responsible for the parasympathetic control of the majority of abdominal viscera. ${ }^{(4)}$

One of the possible causes of altered visceral mobility may be related to functional dyspepsia. This dysfunction is characterized by discomfort or pain with no obvious organic cause which can be identified by endoscopy, having as possible causes abnormal gastroduodenal motility and failure in the accommodation of the stomach - that is, inability of the stomach to properly distend. ${ }^{(6)}$ 
Although no clear evidence has been found between visceral mobility and cervical pain, this study considered the hypothesis that nociceptive excitation due to modifications in the functioning and movement of abdominal viscera may also be one of the causes of NS-NP, and that inhibition of this nociceptive stimulus may result in improvement in NS-NP symptoms. To test this hypothesis, we used manual techniques of visceral manipulation (MV) for the treatment of two NS-NP subjects with functional dyspepsia.

MV was used as it is a therapeutic resource considered capable of improving mobility ${ }^{(4,7,8)}$ and functions ${ }^{(4)}$ of viscera with altered movements. Therefore, a quantitative analysis of two cases ${ }^{(9)}$ was conducted in order to verify the immediate and seven-day postintervention effects of visceral manipulation to the stomach and liver through neck pain, cervical range of motion, and electromyographic activity of the upper trapezius muscle in two NS-NP subjects with functional dyspepsia.

\section{METHODS}

\section{Participants}

Two symptomatic individuals were recruited from a convenience sample of students at the University Nove de Julho. Case A was an 18-year-old female student with a complaint of non-specific neck pain for one year, with reported pain on waking, momentary intermittent pain, and occasional symptoms of paresthesia in the upper limbs. Case B was a 25-yearold female student with a complaint of cervical pain for one year, accompanied by pain in the unilateral temporomandibular joint and medial thoracic region. Both cases presented functional dyspepsia according to the Diagnostic Criteria for Functional Gastrointestinal Disorders Rome III. ${ }^{(10)}$ The Neck Disability Index was used to verify the disability associated with the cervical pain condition, resulting in scores of 15 and 13 for cases A and B, respectively. The present study was approved by the Human Research Ethics Committee of the University Nove de Julho (research protocol $\mathrm{n}^{\mathrm{o}}:$ 1.463.480). All participants/guardians were properly informed regarding the objectives and procedures, and signed a statement of informed consent prior to testing.

\section{Assessment}

The following measurement instruments were used to evaluate the effects of MV: i) Numerical Rating Scale (NRS) to evaluate neck pain;(11) ii) flexiometer (Sami ${ }^{\circledR}$, São Paulo, Brazil, L-6010) to verify cervical range of motion (ROM); iii) Electromyographer (Band pass filter: $20-500 \mathrm{~Hz}$, amplifier gain of 1000, CRMR <120dB - EMG830C [EMG System do Brasil Ltda ${ }^{\circledR}$, Sao Jose dos Campos, Brazil]) to analyze activity of the UT muscle. The surface electromyographic (sEMG) signals were acquired and processed using a 16-bit analog-to-digital converter, with a sampling frequency of $2 \mathrm{kHz}$.

\section{Intervention}

\section{Visceral Manipulation}

Participants were instructed to lie down comfortably on a stretcher in the supine position, with lower limbs flexed and abdomen exposed, and the therapist was positioned to the right side of the patient. The therapeutic intervention began with the left hand of the therapist in contact with the lower region of the stomach, to which a force was applied so that the organ was moved in an upper and lateral left direction, while the right hand controlled and directed the knees of the patient to the right side until the moment when the therapist noticed an increase in tension in the stomach region (Figure 1A). For the liver manipulation, the same procedures were followed; however, the therapist and hand positions were reversed, with contact in the right epigastric region and the knees directed to the left side. The same position was maintained for each organ treated until the therapist could feel, through touch, a decrease in the tension of the viscera (Figure 1B). The mean treatment time was 5 min.

\section{Procedures}

Data were collected only after obtaining informed written consent. Subjects were evaluated before, immediately, and seven days after termination of MV treatment. Figure 1 presents the sequence used for data collection.

The participants were instructed to remain seated in a chair and data on pain and cervical ROM were collected at baseline (T1). Prior to starting the MV treatment, the patient was placed in an instrumentation chair with two force transducers, which allowed appropriate adjustment according to the height of the shoulder of each volunteer (Figure 2). The force signals obtained by the transducers were collected, together with the sEMG signal, by the same signal acquisition system. For sEMG signal collection, a linear array of 10 electrodes (silver bar electrodes, $5 \mathrm{~mm}$ long, $1 \mathrm{~mm}$ diameter, $5 \mathrm{~mm}$ interelectrode distance) was positioned $2 \mathrm{~cm}$ lateral to the midpoint of the line between the $\mathrm{C} 7$ spinous process and the acromion. ${ }^{(12)}$ The EMG signals were recorded on the side with the greatest self-reported pain.

After electrode fixation, the subjects were instructed to perform three shoulder elevations in maximal isometric voluntary contraction (MIVC) against the resistance of the force transducers for $5 \mathrm{~s}$, with an interval of 2 min between collections. Verbal encouragement was given during all MVICs. The maximum peak force between force collections 


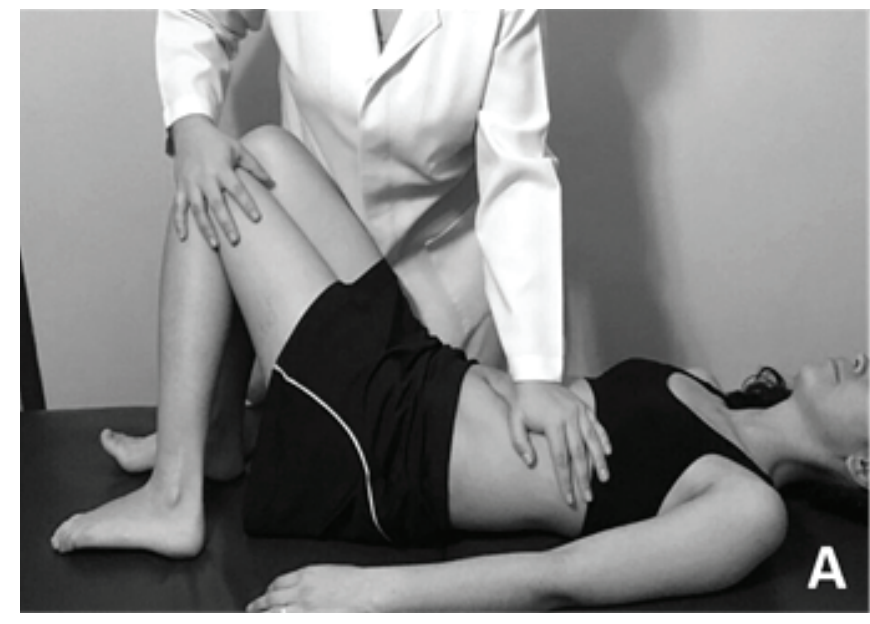

FIGURE 1. Visceral manipulation in stomach (A) and liver (B).

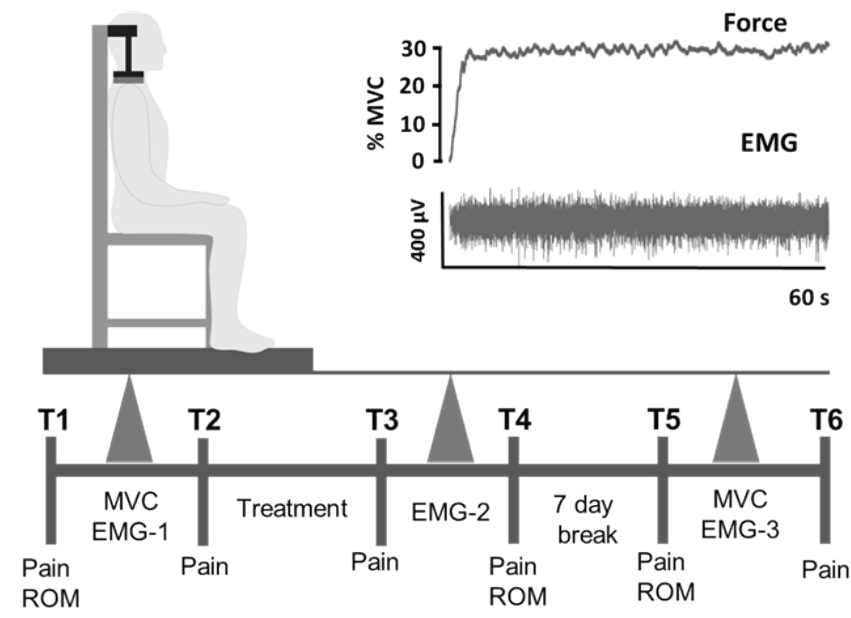

Figure 2. Flow sequence diagram of data recording. EMG $=$ electromyography; $T 1$ = before visceral manipulation (baseline); $T 2=$ after EMG recording; T3 = immediately post visceral manipulation; T4 = after EMG recording; T5 = seven days after the visceral manipulation; $R O M=$ range of motion, $T 6=$ after $E M G$ recording.

(Newtons) was considered as $100 \%$ of MIVC. A $30 \%$ MIVC training line was established as feedback on the computer screen, and subjects were instructed to maintain shoulder elevation over this training line for $65 \mathrm{sec}$ (EMG-1). After a 2-min rest interval, data on pain were collected (T2). Subsequently, treatment with MV was started. After a 5-min rest interval, new evaluations of pain and cervical ROM were performed (T3), followed by a new sEMG signal collection (EMG-2) in the same manner as performed during EMG-1. Data on pain were collected after a 2-min rest interval (T4). After a period of seven days, a further evaluation of pain and cervical ROM (T5) was performed, followed by sEMG signal collection as performed during EMG-1 (3 MIVC initially and shoulder elevation over the training line with $30 \%$ MVIC for 60 s) (EMG-3). After a 5-min rest interval,

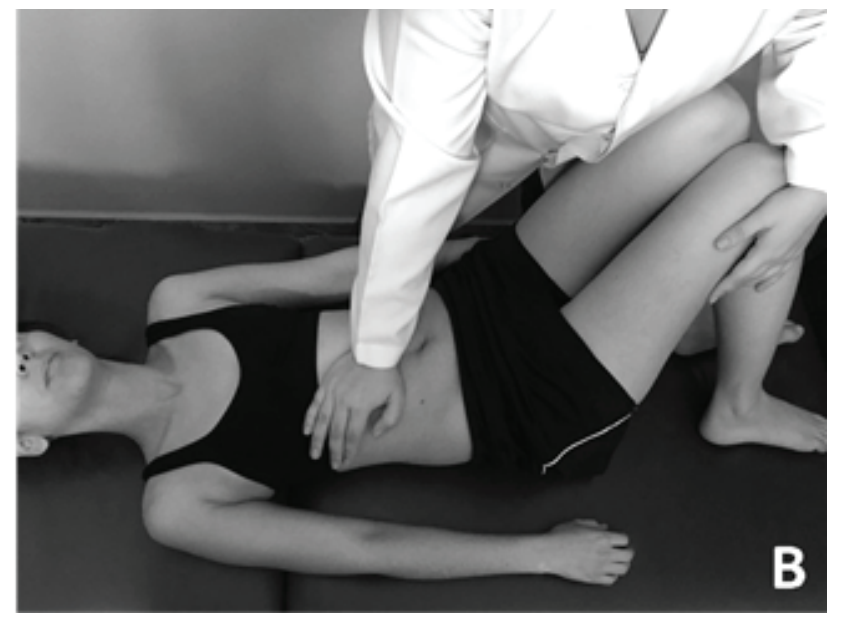

data on pain were collected (T6). All participants received training prior to the shoulder elevations, based on the previously determined force levels

\section{EMG Signal Processing}

For the amplitude analysis of the sEMG signals, the first $5 \mathrm{~s}$ of the signal were discarded and the root mean square (RMS) was calculated using a $1 \mathrm{~s}$ moving window ( 60 values in total) for each of the 8 signals captured by the array electrode. The mean of all calculated RMS values was considered as global RMS (RMSG). The estimated muscle fiber conduction velocity (MFCV) ${ }^{(13)}$ was obtained for every $5 \mathrm{~s}$ of the EMG signal and the mean values calculated. EMG signals were processed by performing specific routines with MATLAB software (version R2010a; The MathWorks Inc., Natick, MA, USA).

\section{RESULTS}

The results obtained pre (baseline), immediately post, and seven days after the MV intervention are presented from the cervical ROM and EMG signal in Table 1. The results observed in the two subjects (A and $\mathrm{B}$, respectively) demonstrated a general increase in cervical ROM (range: $12.5 \%$ to $44.44 \%$ ) and amplitude of the EMG signal (immediately postintervention: 57.62 and 20.78; post seven days: $53.54 \%$ and $18.83 \%$ ) and an increase in muscle fiber conduction velocity immediately postintervention $(4.44 \%$ and $7.44 \%)$ and a decrease seven days postintervention $(25.25 \%$ and $21.18 \%)$,

In the analysis of pain intensity, the mean values obtained in assessments T1 and T2 (Mean NRS: A $=6.5, \mathrm{~B}=6.5$ ), were considered as pretreatment values, while the mean values from assessments $\mathrm{T} 3$ and T4 (Mean NRS: $\mathrm{A}=5, \mathrm{~B}=1.5$ ) as immediately posttreatment values, and the mean from assessments 
TABLE 1. Cervical Range of Motion (ROM) and Muscle Activation Data (EMG)

\begin{tabular}{lcccccc}
\hline & \multicolumn{3}{c}{ Case A } & \multicolumn{3}{c}{ Case B } \\
\hline Cervical ROM & T1 & T3 & T5 & T1 & T3 & T5 \\
Flexion $\left(^{\circ}\right)$ & 51 & 55 & 58 & 50 & 50 & 60 \\
Extension $\left(^{\circ}\right)$ & 10 & 18 & 12 & 30 & 35 & 30 \\
Right lateral & 28 & 30 & 42 & 20 & 25 & 30 \\
flexion $\left(^{\circ}\right)$ & & & & & & \\
Left lateral & 20 & 25 & 36 & 20 & 28 & 25 \\
flexion $\left(^{\circ}\right)$ & & & & & & \\
EMG & $E M G-1$ & $E M G-2$ & $E M G-3$ & $E M G-1$ & $E M G-2$ & $E M G-3$ \\
RMS $(\mu \mathrm{V})$ & 24.71 & 38.95 & 37.94 & 34.71 & 41.95 & 41.27 \\
MFCV $(\mathrm{m} / \mathrm{s})$ & 4.95 & 5.17 & 3.70 & 5.24 & 5.63 & 4.13 \\
\hline
\end{tabular}

$\mathrm{T} 1$ = before visceral manipulation (baseline); $\mathrm{T} 3=$ immediately post visceral manipulation; $\mathrm{T} 5=$ seven days after the visceral manipulation; $\mathrm{MFCV}=$ muscle fiber conduction velocity; $\mathrm{RMS}=$ root mean squared; $\mathrm{EMG}=$ electromyography.

T5 and T6 (Mean NRS: $\mathrm{A}=0, \mathrm{~B}=0$ ) as seven-day postintervention values. The results demonstrated a decrease in neck pain (immediately postintervention: $23.07 \%$ and $76.92 \%$; post seven days: $100 \%$ for both subjects)

\section{DISCUSSION}

On the whole, the results of this study reinforce the hypothesis that nociceptive stimuli resulting from modifications in the functioning and/or movement of abdominal viscera may have a relation with cervical pain. In addition, some previously described results on the clinical effects of MV interventions ${ }^{(14-16)}$ increase the evidence that, in many cases, musculoskeletal pain may have its origin in a nociceptive stimulus generated by a visceral alteration.

As examples of this possible relation between muscular pain from viscera with or without hyperalgesia, the following are highlighted: trophic alterations in the superficial and deep paraspinal muscles in subjects with gallbladder disfunction, (14) increase in the pressure pain threshold of the L1 paraspinal muscles after manipulation of the sigmoid colon, ${ }^{(15)}$ and decreased mobility of the right kidney and bladder in subjects with nonspecific low back pain. ${ }^{(8)}$

A possible relation between visceral alterations and musculoskeletal pain may be in the viscerosomatic convergence that occurs between parts of the dorsal horn neurons of the thoracolumbar medulla. In general, these neurons are responsible for the visceral and somatic afferents (muscles, skin, joints); however, when they receive a nociceptive stimulus from a viscera, they can promote a decrease in the activation threshold at the medullary level, ${ }^{(17,18)}$ which may contribute to increased sensitivity to pain and, as a consequence, generate a spasm of the muscles corresponding to the medullar level that presents the facilitation.

However, in the present study, it is possible that this afference did not occur through the visceral sensory pathways since medullary facilitation may have been provided by the phrenic nerve, which innervates the diaphragmatic muscle, the subdiaphragmatic peritoneum, ${ }^{(3)}$ coronary ligaments, falciform and capsule of the liver. ${ }^{(4)}$ In this case, a decrease in the mobility of the viscera located below the diaphragm muscle could impair its function and, thus, generate afferent stimuli to the phrenic nerve. Thus, the reduction in cervical pain and alterations in the sEMG signal found in the present study strengthen the possibility that the MV has an afferent stimulus from the manipulated organs.

In addition, another important factor to be considered as an explanation for the results found in the present study is that the cervical and abdominal regions have important mechanical relationships, which occur through a fascial pathway. Thus, an alteration in the mobility of the peritoneum or diaphragm could have repercussions on fascia present in the cervical region. ${ }^{(19,20)}$ This anatomical relationship should also be considered as a hypothesis that NS-NP may be related to a cervical mechanical alteration caused by the loss of mobility of fascia related to abdominal viscera.

The increase in amplitude (RMS) and decrease in MFCV seven days after intervention, in the two cases studied, as well as the decrease in pain, confirm the positive effects of the MV technique used in the present study. In general, subjects with NS-NP present higher MFCV of the UT muscle in relation to healthy individuals. ${ }^{(21)}$ This observation contributes to the argument that the decrease in the neural MFCV observed in these subjects is due to the decrease in pain. This clinical condition may also have contributed to the increase in the sEMG signal amplitude, as already observed in NS-NP subjects treated with acupuncture. (22)

In general, it has been shown that pain promotes a decrease in the electrical activity of the UT muscle during an isometric contraction. ${ }^{(23,24)}$ However, this clinical condition alone may not be enough to explain the muscular electrophysiological changes. ${ }^{(25)}$ These alterations are most likely a combination of changes in neural input and muscle properties. ${ }^{(25-27)}$ These observations once again reinforce the possibility that visceral alterations may produce nociceptive input capable of promoting changes in the threshold of muscle activation at the spinal cord level and, consequently, alterations in the pattern of activation of the muscles corresponding to the affected medullar level.

There are some relevant limitations of this study that might be important for future discussions about this theme. First, only a single visceral manipulation intervention was applied, without a previous visceral mobility evaluation, and not associated with a long-term treatment, and the case reports included the results of only two subjects without any control 
group. Trials with larger samples are necessary to confirm these positive results. Second, in this study, the standard error measurement was not calculated for data obtained through the sEMG. Thus, it is not possible to state that the sEMG data could not have been influenced by the methodological design of this study. However, it is important to note that the changes found in the cervical ROM and NRS also demonstrated that MV improved neck pain and cervical mobility.

As NS-NP is considered a disorder with multifactorial causes, there are still many mechanisms that can be explored. Thus, as previously reported, the results demonstrated in the present study suggest that alterations in the mobility of abdominal viscera have influence on pain and cervical mobility, as well as on the sEMG signal of the UT. However, further studies are necessary to allow better understanding of what physiological mechanisms work in this relationship between visceral mobility and musculoskeletal pain.

\section{CONCLUSION}

In the present case study it was observed that visceral manipulation seemed to reduce pain, increase cervical mobility and electromyographic activity, and decrease muscle fiber conduction velocity of the upper trapezius muscle in two non-specific neck pain subjects with functional dyspepsia.

\section{ACKNOWLEDGMENTS}

This study is supported by the Universidade Nove de Julho (UNINOVE, Brazil) and the Brazilian fostering agency Fundação de Amparo a Pesquisa (FAPESP: Process $n^{\circ}$. 2013/ 13839-9) and Coordination for the Improvement of Higher Education Personnel (CAPES: Process $\left.n^{\circ} 1712107\right)$.

\section{CONFLICT OF INTEREST NOTIFICATION}

The authors report no conflicts of interest. The authors are wholly responsible for the content of the article.

\section{COPYRIGHT}

Published under the CreativeCommons AttributionNonCommercial-NoDerivs 3.0 License.

\section{REFERENCES}

1. Merskey H, Bogduk N. Classification of Chronic Pain: Descriptions of Chronic Pain Syndromes and Definitions of Pain Terms. Seattle, WA: IASP Press; 1994.
2. Binder A. The diagnosis and treatment of nonspecific neck pain and whiplash. Eur Medicophysica. 2007;43(1):79-89.

3. Strandring S. Gray's Anatomy, 41st ed. Amsterdam, The Netherlands: Elservier; 2016.

4. Barral J-P, Mercier P. Visceral Manipulation, rev. ed. Seattle, WA: Eastland Press; 2006.

5. Shoja MM, Oyesiku NM, Griessenauer CJ, Radcliff V, Loukas $\mathrm{M}$, Chem JJ, et al. Anastomoses between lower cranial and upper cervical nerves: a comprehensive review with potential significance during skull base and neck operations, Part I: trigeminal, facial, and vestibulocochlear nerves. Clin Anat. 2014;27(1):118-130.

6. Brun R, Kuo B. Functional dyspepsia. Ther Adv Gastroenterol. 2010;3(3):145-164.

7. Markel Feldt C, Carriere B. The Pelvic Floor, 1st ed. New York: Thieme Medical Pub; 2006.

8. Tozzi P, Bongiorno D, Vitturini C. Low back pain and kidney mobility: local osteopathic fascial manipulation decreases pain perception and improves renal mobility. J Bodyw Mov Ther. 2012;16(3):381-391.

9. Hymel GM. Research Methods for Massage and Holistic Therapies. Mosby's Massage Career Development Series. St. Louis, MO: Elsevier Mosby; 2006.

10. Rome Foundation. Guidelines-Rome III Diagnostic criteria for functional gastrointestinal disorders. J Gastrointest Liver Dis. 2006;15(3):307-312.

11. Ferreira-Valente MA, Pais-Ribeiro JL, Jensen MP. Validity of four pain intensity rating scales. Pain. 2011;152(10): 2399-2404.

12. McLean L, Chislett M, Keith M, Murphy M, Walton P. The effect of head position, electrode site, movement and smoothing window in the determination of a reliable maximum voluntary activation of the upper trapezius muscle. J Electromyogr Kinesiol. 2003;13(2):169-180.

13. Farina D, Merletti R. Methods for estimating muscle fibre conduction velocity from surface electromyographic signals. Med Biol Eng Comput. 2004;42(4):432-445.

14. Giamberardino MA, Affaitati G, Lerza R, Lapenna D, Costantini R, Vecchiet L. Relationship between pain symptoms and referred sensory and trophic changes in patients with gallbladder pathology. Pain. 2005;114(1-2):239-249.

15. McSweeney TP, Thomson OP, Johnston R. The immediate effects of sigmoid colon manipulation on pressure pain thresholds in the lumbar spine. J Bodyw Mov Ther. 2012;16(4): 416-423.

16. Vecchiet L, Vecchiet J, Giamberardino MA. Referred muscle pain: clinical and pathophysiologic aspects. Curr Rev Pain. 1999;3(6):489-498.

17. Cervero F, Jänig W. Visceral nociceptors: a new world order? Trends Neurosci. 1992;15(10):374-378.

18. Euchner-Wamser I, Sengupta JN, Gebhart GF, Meller ST. Characterization of responses of T2-T4 spinal cord neurons to esophageal distension in the rat. $J$ Neurophysiol. 1993;69(3):868-883.

19. Bordoni B, Zanier E. Anatomic connections of the diaphragm: influence of respiration on the body system. $J$ Multidiscip Healthc. 2013;6:281-291.

20. Paoletti S. The Fasciae: Anatomy, Dysfunction and Treatment, 1st ed. Seattle, WA: Eastland Press; 2006. 
21. Falla D, Farina D. Muscle fiber conduction velocity of the upper trapezius muscle during dynamic contraction of the upper limb in patients with chronic neck pain. Pain. 2005;116(12):138-145.

22. Silva AC de O, Biasotto-Gonzalez DA, Dos Santos DM, De Melo NC, Fidelis de Paula Gomes CA, Ferreira Amorim C, Politti F. Evaluation of the immediate effect of auricular acupuncture on pain and electromyographic activity of the upper trapezius muscle in patients with nonspecific neck pain: a randomized, single-blinded, sham-controlled, crossover study. Evid-Based Complement Altern Med. 2015;2015:523851.

23. Ge H-Y, Arendt-Nielsen L, Farina D, Madeleine P. Gender-specific differences in electromyographic changes and perceived pain induced by experimental muscle pain during sustained contractions of the upper trapezius muscle. Muscle Nerve. 2005;32(6):726-733.

24. Madeleine P, Leclerc F, Arendt-Nielsen L, Ravier P, Farina D. Experimental muscle pain changes the spatial distribution of upper trapezius muscle activity during sustained contraction. Clin Neurophysiol. 2006;117(11):2436-2445.
25. Falla D, Farina D. Neuromuscular adaptation in experimental and clinical neck pain. J Electromyogr Kinesiol. 2008;18(2):255-261.

26. Kadi F, Waling K, Ahlgren C, Sundelin G, Holmener G, ButlerBrowne GS, Thornell L-E. Pathological mechanisms implicated in localized female trapezius myalgia. Pain. 1998;78(3): 191-196.

27. Lindman R, Hagberg M, Angqvist KA, Söderlund K, Hultman E, Thornell LE. Changes in muscle morphology in chronic trapezius myalgia. Scand J Work Environ Health. 1991;17(5):347-355.

Corresponding author: Fabiano Politti, PT, PhD, Postgraduate Program in Rehabilitation Sciences, Physical Therapy Department, Universidade Nove de Julho, Rua Vergueiro, 235 - Liberdade, São Paulo 01504-001, SP, Brazil

E-mail: fabianopolitti@gmail.com 